\title{
Automated Vision-Based Force Measurement of Moving C. elegans
}

\author{
Ali Ghanbari, Volker Nock, Richard Blaikie, Senior Member, IEEE, XiaoQi Chen, Senior Member, \\ IEEE, J. Geoffrey Chase, Senior Member, IEEE, and Wenhui Wang*, Member, IEEE
}

\begin{abstract}
- this paper reports an automated tracking and force measurement system for $C$. elegans in motion, based on microscopy computer vision. A polydimethylsiloxane (PDMS) device was microfabricated to allow $C$. elegans to move in a matrix of micropillars in a channel. An image processing algorithm has been developed to track the worm and deflecting micropillars automatically in purpose of quantifying multipoint locomotive forces of a moving $C$. elegans. The developed system is able to visually resolve force with a $0.33 \mu \mathrm{N}$ resolution. A maximum force level of $55.41 \mu \mathrm{N}$ for a young adult wild type $C$. elegans was observed.
\end{abstract}

\section{INTRODUCTION}

$\mathrm{C}$ TAENORHABDITIS elegans (C. elegans), a soildwelling multicellular eukaryotic nematode, is widely used as a model organism for studies in cellular differentiation, neural networking and molecular genetics due to its relative simplicity in anatomy. With a fully sequenced genome [1] and favorable gestation times, the semi-transparent $C$. elegans has been successfully established as an experimental genetic system regarding the relationship between genes and locomotive behavior [2]. In a normal environment, nematodes like $C$. elegans, exhibit a sinusoidal movement pattern induced by waves of muscle contraction and local bending of the cuticle [3]. Changes in the locomotive behavior of the nematodes can be induced by natural aging [4], structured environment, external exposure to toxins and drugs [5-7], or through the manipulation of specific genes [8-11].

Genetic modification in particular can be used to yield $C$. elegans mutants with different numbers of muscle arms, which [12] are physical connections established between $C$. elegans muscles and the motor neurons via membrane extensions. As muscle arms function as paths for muscles to receive stimulation from the nerve, their number is most likely to affect the motion pattern of the nematode. Determining the correlation between muscle arms and motion patterns can therefore be of specific implications in identifying the role of individual genes in locomotion, through the phenotypic locomotive behavior study. To conduct such a study, a force sensor suitable for the small animal is required. As the nematode is tiny $(\sim 1 \mathrm{~mm}$ in length

A. Ghanbari, X. Chen, J. G. Chase and W. Wang are with the Department of Mechanical Engineering, University of Canterbury, Private Bag 4800, Christchurch 8140, New Zealand (corresponding author e-mail: wenhui.wang@canterbury.ac.nz).

V. Nock and R. Blaikie are with MacDiarmid Institute for Advanced Materials and Nanotechnology and the Department of Electrical and Computer Engineering, University of Canterbury, Private Bag 4800, Christchurch 8140, New Zealand. and $<90 \mu \mathrm{m}$ in width, invisible to the naked eye), the typical sensors used in millimeter and larger scales are unable to detect the micro-Newton-level force.

Two decades ago, the flexible substrate method was introduced for characterizing mechanical interactions between biological cells and their surrounding environment [13]. More recently, an innovative alternative approach employing microfabricated polydimethylsiloxane (PDMS) post/pillar structures as force transducers was reported to visually measure traction forces generated by adherent cells (smooth muscle cells [14], epithelial cells [15], and cardiac myocytes [16]). Unlike flexible thin substrates, the post/pillar structure does not require heavily complex computations for calculating traction forces. Compared to silicon-based MEMS devices, PDMS-based devices are biocompatible and can be readily fabricated using softlithography techniques [17].

There have been few systems for measuring forces of $C$. elegans. A silicon piezoresisitve cantilever [3] has been fabricated to characterize the mechanics of $C$. elegans body tissue. Constrained onto its culture surface or a testbed by the cantilever free end, the worm is not able to move freely, which excludes the sensor from sensing the dynamic force of moving C. elegans. Very recently, a device with SU-8 based rising pillars [18] has been developed to measure dynamic forces, with each pillar having four 90-degree-spaced gold resistors as strain gauges, which were deployed on its bottom base. The resistance change of the strain gauges gives the force applied at the tip of the pillar. Three main drawbacks exist for this device: (i) use of the strain gauges complicates the microfabrication procedures and measuring instrumentation; (ii) heat dissipated from the gold resistors is likely to affect the natural locomotion of the temperaturesensitive nematode; (iii) complicated structure of the device easily occludes a moving worm, causing unwanted barriers for vision-based worm locomotion observation.

To overcome these drawbacks, a simpler force sensor is highly desired. Continuing from previous works [19], [20], this paper reports on a micropillar-based on-chip system capable of constantly measuring force of moving C. elegans. The system consists of a micropillar-based device made of PDMS only (Fig. 1a) and an image processing algorithm for resolving force from the deflection of the cantilever-like pillar. The microdevice and automated visual tracking of pillar deflection form an integrated powerful system for measuring dynamic forces of moving C. elegans with a high resolution. In addition to dynamic force, the neat structure 

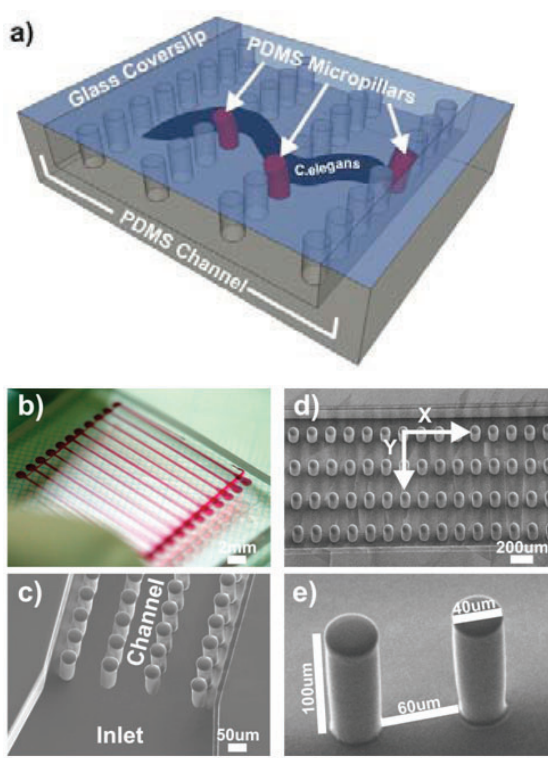

Fig. 1. PDMS micropillar-based device for C. elegans force measurement. (a) The schematic of a worm deflecting micropillars while moving between them in a channel. (b) Photograph of the device with multiple channels having an inlet and outlet on both ends. The channels are filled with dye-coloured water for illustration. (c) Zoom-in view of one end of the channel with pillars and inlet. (d-e) SEM micrographs of the pillars with dimensions labeled.

and transparency of the device allow other locomotive metrics (e.g., speed, distance, wave amplitude) [21] to be collected by developed automated visual tracking system, which will lead to a unique on-chip phenotypic screening framework for small animals like C. elegans.

\section{II.MICROPILLAR-BASED PDMS DEVICE}

The device consists of a number of straight and parallel channels (those filled with dye-colored water in Fig. 1b). Each channel, containing several parallel rows of rising cantilever-like micropillars supported by the channel base, functions as an independent force measuring unit dedicated for one individual C. elegans. By doing so, cross-contamination of worms which otherwise share a same channel is eliminated. In the mean time, to increase fabrication throughput, multiple channels are designed in one chip to allow more worms to be assayed. More importantly, due to the same fabrication procedure, negligible variances in micropillars (size and mechanical property) makes force measurements highly consistent and comparable across channels or worms.

A glass coverslip is put on top of the PDMS device to protect the micropillars, worms, and channels from potential physical contamination, interference or damage. To facilitate loading of solutions, water, or the worm to the enclosed micropillar-filled channel, an inlet (Fig. 1c) and outlet are extended from both ends of each channel, which are simply an open and clear concaved planar area. To prevent the micropillars from sticking to the glass coverslip, the depth of the channel is set to $20 \mu \mathrm{m}$ greater than the height of micropillars, leaving a vertical gap between the top of the pillar and the coverslip.
Currently, the number of rows per each channel is four but more rows of micropillars can be easily added to the device as necessary. According to the width of an adult $C$. elegans, the height of pillars is $100 \mu \mathrm{m}$, to ensure the worm moves inside the arrays of pillars rather than on their top. The diameter of pillars can affect the force measurement resolution and currently is set to be $40 \mu \mathrm{m}$. The inter-pillar distances in the $x$ - and $y$-directions are 60 $\mu \mathrm{m}$ and $110 \mu \mathrm{m}$, respectively. Fabrication of the device was described previously in [19] and thus omitted.

Prior to loading worm, the surface of the device was rendered hydrophilic by use of a laboratory corona treater (Electro-Technic Products). The top of the device was then covered with a standard $22 \times 22 \mathrm{~mm}$ glass coverslip (ESCO Inc.) to enclose the channel part containing the micropillar array. Following this, a small drop of deionized water (DI) was dispensed onto the uncovered inlet by pipette, moisturizing the channel via diffusion. This pre-loading of the device with water provides the moisturized environment required for the worm to move naturally. However, to minimize the effect of fluids on $C$. elegans motion, the amount of water was critically controlled so that only a very thin layer of water with a negligible thickness relative to the worm width exists throughout the channel.

\section{FORCE SENSING PRINCIPLE}

Fig. 2 shows the deflection of a pillar undergoing force which is originated from the contact of the worm body with the pillar. Through an imaging system (a camera mounted on a microscope), the deflection $\Delta$ of the free end of the pillar can be recorded and measured by developed image processing algorithm. With the deflection available, the force $f$ is subsequently obtained by a linear spring forcedeflection model

$$
f=k \Delta
$$

where $k$ is the stiffness of the pillar.

As the worm moves inside the array of pillars, the force does not apply at the free end of the pillar, which makes the total deflection of the free end is attributed to two parts: (i) the deflection $\delta$ proportional to the load force at the force loading point $A$, and (ii) the linear displacement $\delta^{\prime}$ of the free end $B$ geometrically transmitted from point $A$.

A force-deflection model has been derived and reported in [19], which gives the stiffness $k$ as follows:

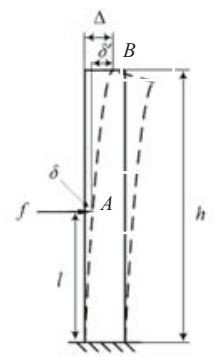

Fig. 2. Schematic of the bending pillar for force analysis. $h$ is length of the pillar, $f$ the force, $l$ the height where force is imposed, $\delta$ deflection at force point, $\delta$ ' the linear displacement of the free end due to $\delta$, and $\Delta=\delta$ $+\delta$ ' total deflection at the free end of the pillar. 


$$
k=\left\{\left(\frac{l^{3}}{3 E I}+\frac{d^{2}(1+\gamma) l}{4 E I}\right)+\frac{l^{2}}{2 E I}(h-l)\right\}^{-1}
$$

where $d$ is the pillar diameter, $l$ is the length from the load to the support, $I$ is the moment of inertia, and $E$ and $\gamma(=0.5)$ are Young's modulus and Poisson's ratio for PDMS, respectively. Based on fabrication specifications, Young's modulus $(E)$ of the PDMS is equal to $2.6 \mathrm{MPa}$ [18].

Note that Eq. (1) implies that the direction of load follows that of the deflection, permitting the dynamic force of a moving worm to be resolved with both magnitude and direction.

\section{AutOMATED VISUAL MEASUREMENT OF PILLAR DEFLECTION}

An image processing algorithm was developed to detect and track those pillars which are in contact with $C$. elegans moving in array of pillars, and measure their deflection all automatically. Fig. 3 shows the top view of $C$. elegans moving inside micropillars, where the deflection of those pillars with C. elegans force loads is visually noticeable. For each pillar subject to deflection, top circular surface could be fitted a circle to detect the circular center position. The deflection is equivalent to the displacement of the circle center with respect to its neutral position where the load is zero. The developed algorithm processes stream of image frames in four main steps, namely, identifying contact pillars and worm, detecting circles for contact pillars, determining neutral position for contact pillars, and tracking pillars.

\section{A. Detecting Contact Pillars and Worm}

Pillars of interest for force measurement purposes are those that worm contacts and would force against. Thus, it is necessary to separate non-contact pillars from contact pillars for next steps to improve accuracy and efficiency.

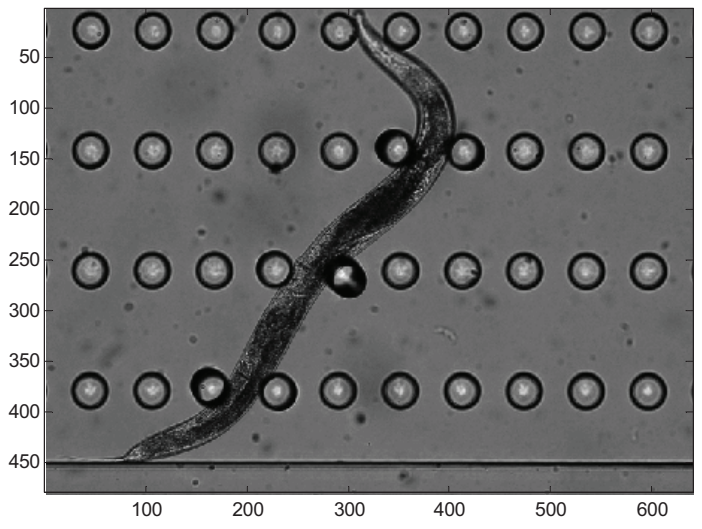

Fig. 3. A raw image frame showing worm inside a matrix of pillars.

As Fig. 3 indicates, all non-contact pillars are isolated from either the worm body or neighboring pillars. In contrast, contact pillars are mixed together with C. elegans, forming the largest object in the image. This is generally true for all image frames and results in an algorithm to differentiate non-contact pillars and contact ones. The detailed steps follow.

1. The gray-scale raw image is binarized with an optimized threshold empirically found to give the best result for subsequent steps. Fig. 4a shows the resultant binary image.

2. An algorithm based on morphological reconstruction [22] was applied to fill in holes in the binary image. A hole is a block of black pixels enclosed by a block of connected white pixels. This step aims at labeling all objects with white pixels. Fig. $4 \mathrm{~b}$ shows resultant image with holes filled.

3. Counting the number of white pixels of each isolated object, the object with the largest number is taken as the combination of worm and the contact pillars. Fig. $4 \mathrm{c}$ shows the resultant image with all non-contact pillars removed, indicating the non-contact pillars' coordinates can be determined.

a)
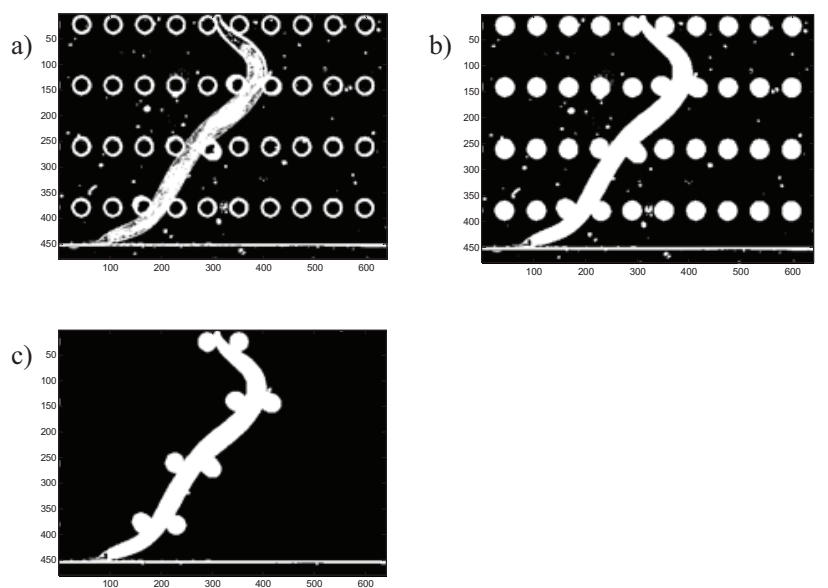

Fig. 4. Noncontact pillars elimination process. (a) Converting to binary image. (b) Filling holes. (c) Detected contact pillars and worm.

\section{B. Detecting Circles for Contact Pillars}

As their name indicates, contact pillars are partially overlapped or occluded by the worm body. To separate these circular pillars from the worm body, a circle detection algorithm based on Hough transform [23] and array of accumulators [24] was adapted for this purpose. Before Hough transform, the raw image is processed with the Sobel operator only in the area consisting of contact pillars and worm, rather than in the entire image. The resultant gradient image is then used in Hough transform for circle detecting.

A circle in the 2-D $x-y$ image plane (also called as geometric space) can be defined as

$$
(x-a)^{2}+(y-b)^{2}=r^{2}
$$

$\{a, b, r\}$ is a triplet defining the center and radius of the circle. Hough transform suggests that any point $\left(x_{i}, y_{i}\right)$ in geometric space could be transformed onto a conical surface in the $a-b-r$ parameter space described by

$$
\left(x_{i}-a\right)^{2}+\left(y_{i}-b\right)^{2}=r^{2}
$$

Therefore, each 2-D point is mapped to a 3-D right circular cone surface. If any two cones in the parameter space intersect at $\left\{a_{0}, b_{0}, r_{0}\right\}$, their corresponding points in 
geometric space lie on the same circle characterized by this triplet [25]. In implementation, a 3-D array of accumulators representing the 3-D parameter space could be defined. For those points on the perimeter of a same circle, a triplet will correspond to the accumulation cell where the locally largest number of cone surfaces intersect.

For the device used in this work, the pillars have a known diameter. Thus, in the parameter space, $r$ in Eq. (4) is a constant and a 2-D array of accumulator can be used. Fig. 5 shows the Hough transform array of accumulators for the case shown in Fig. 3. Note there are eight peaks in the graph, corresponding to the eight contact pillars. The $x-y$ coordinates of the peaks correspond to the pillar centers.

Normally, in implementation of the Hough transform, each edge point is mapped to the full spectrum of those discretized accumulator. The computation burden can be lessened by using image gradient of the edge point. In principle, for an edge point $\left(x_{i}, y_{i}\right)$ on a circle $\left\{a_{0}, b_{0}, r_{0}\right\}$, its gradient should point to the circle center [26]. In other words, the center point $\left(a_{0}, b_{0}\right)$ is collinear with $\left(x_{i}, y_{i}\right)$ and the slope of the straight line is given by the gradient. In comparison, without the gradient information, the center point $\left(a_{0}, b_{0}\right)$ should be searched through a circular $(2 \pi)$ range around $\left(x_{i}, y_{i}\right)$. In real computation, therefore, an angle range $\Phi$ around the gradient can be specified to narrow the

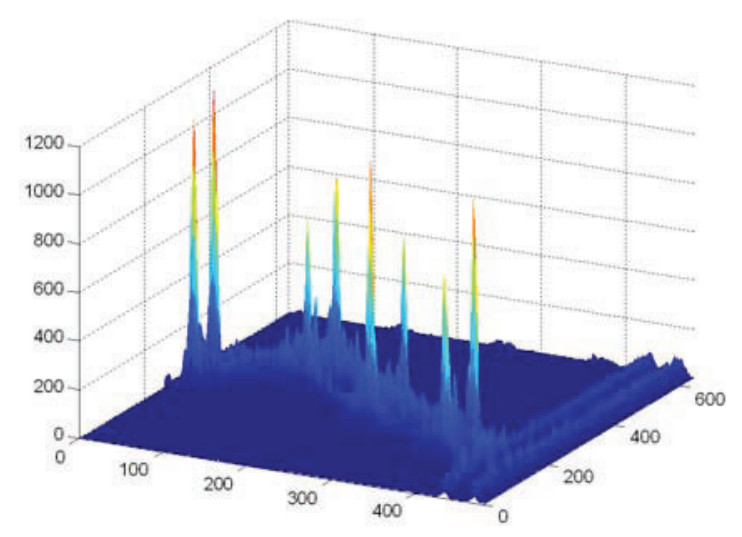

Fig. 5. 3-D view of array of accumulators. Note the $x-y$ coordinates correspond to the image plane. Spikes correspond to circle centers.

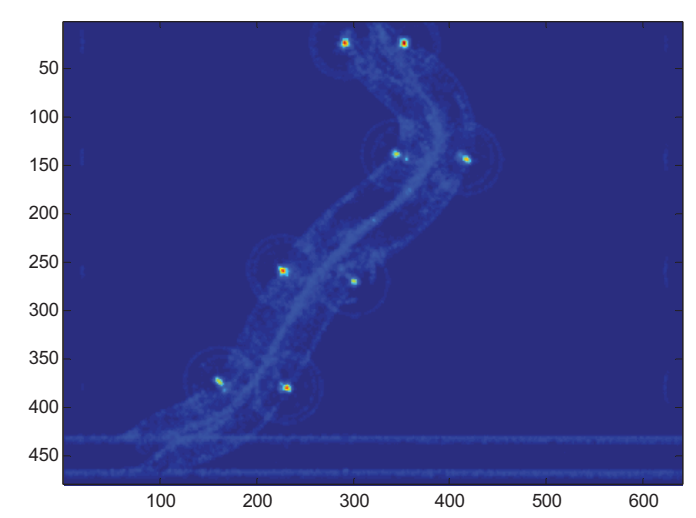

Fig. 6. Top view of array of accumulators for Hough transform for the case shown in Fig. 3. Note here the eight brightest points correspond to the circle centers, $x-y$ coordinates correspond to the image plane.

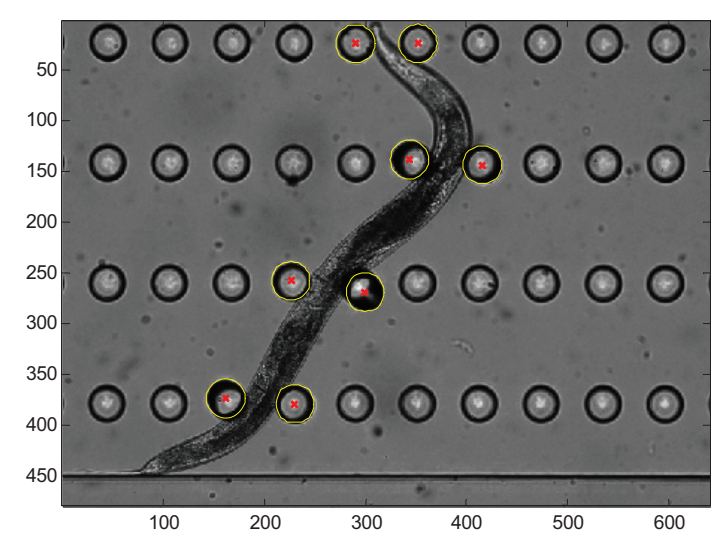

Fig. 7. The eight detected contact pillars (yellow circles) and corresponding centers (red x's) superimposed on the raw image.

searching range of $\left(a_{0}, b_{0}\right)$ in the parameter space. As a result, the computation burden drops by a factor of $2 \pi / \Phi$ [26].

Fig. 7 shows the eight detected circles and centers superimposed on the raw image.

\section{Determining the Neutral Position for Contact Pillars}

For each contact pillar, the deflection caused by worm is equivalent to the displacement of the circle center with respect to its neutral position where the load is zero. The neutral center point of these contact pillars can be interpolated from the center points of neighboring noncontact pillars, by making use of the known geometric parameters (pillars span equidistantly in row and column directions for the device). The Hough transform again can be used to obtain the center points for non-contact pillars, whose region in the image has been determined in Section IV-A.

\section{Tracking Contact Pillars}

From frame to frame, for automated force measurement, it is required to track individual contact pillars so that the algorithm is able to index them correctly. To this end, similar to previous work [20], a template matching algorithm with template update is used to track the motion of the contact pillars. Template matching with constant template update permits small changes (due to worm motion) in image patterns between successive frames of images; therefore, it is capable of robustly tracking the top surfaces of the contact pillars.

Figs 8 and 9 show two sets of four consecutive processed image frames even when microscopy stage was moved along vertical and horizontal axes respectively to bring the worm back to the field of view of the image. Tracking of contact pillars proves to work very well.

\section{C. ELEGANS FORCE MEASUREMENT EXPERIMENT}

\section{A. Experimental Setup and Data Processing}

Wild-type young worm movement through the micropillar arrays was imaged using a Nikon Eclipse 80i fluorescence microscope. A digital camera (DS-5Mc, Nikon) was used to record movies. When worm moved out of the field of 

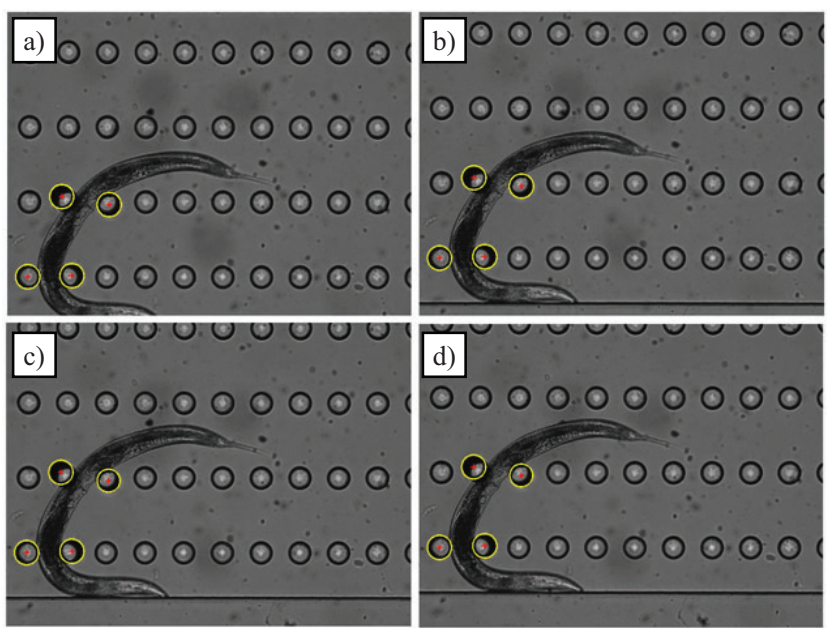

Fig. 8. Four consecutive image frames (a)-(d) with horizontal motion for the microscopy stage. Note the four contact pillars are constantly tracked.

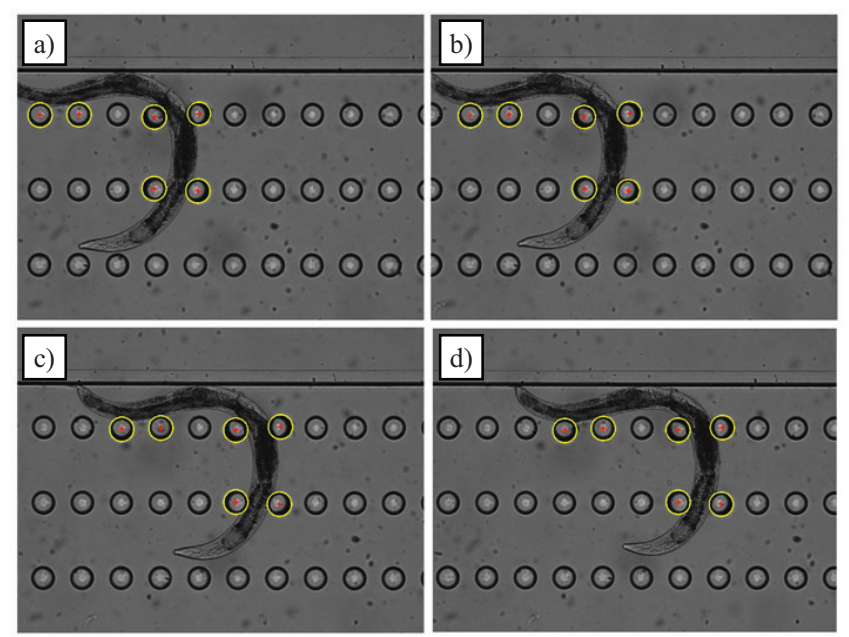

Fig. 9. Four consecutive image frames (a)-(d) with vertical motion for the microscopy stage. Note the six contact pillars are constantly tracked.

view, the stage was manually moved to bring the worm back to the image. Developed image processing algorithm was used to determine pillars deflection, and to resolve corresponding forces with the linear force-deflection model (Eq. (1)).

\section{B. Force Resolution}

For our imaging system with equal pixel size in both $x$ and $y$-directions, force resolution, $\partial f$, is given by the following expression:

$$
\partial f=k \cdot u \cdot \sigma
$$

where $k$ is the stiffness of the pillar given by Eq. (2), $u$ is the pixel size $(1.18 \times 1.18 \mu \mathrm{m} / \mathrm{pixel})$ in either direction, and $\sigma$ is the visual resolution for the pillar deflection $(0.05$ pixel). Corresponding to a worm width of $60 \mu \mathrm{m}$, the stiffness of the pillar is $5.6 \mu \mathrm{N} / \mu \mathrm{m}$, thus the force resolution is $0.33 \mu \mathrm{N}$.

\section{Force Measurement Results and Discussion}

Fig. 10 shows the force measurement of a selected pillar with which the worm had a whole contact interaction (i.e., initial contact, more deflecting, and exit of contact). During this course, the developed force measurement system is capable of constantly tracking the dynamic force trends in $C$. elegans continuous motion.

Two interesting observations were obtained which may have important implications for C. elegans biomechanics and dynamics modeling, although more experiments should be done for conclusive results. One observation was that the force was always normal to the curve of worm motion trajectory, indicating the lateral force is the main thrust, which generates the curvature of the worm body and subsequently the curved motion. The same mechanism exists [27] for a worm moving on an agar surface, which does not have any enhanced structures such as pillars in this study. Interestingly, it implies that the worm maintains its motion generation mechanism by producing lateral thrust regardless of its environment, which can be either natural (i.e., agar surface) or artificially structured such as pillars. This mechanism may be inherent in the muscle contraction of $C$. elegans.

The second observation was that when the worm forwarded, different body parts generated randomly varying force levels for motion. This suggests that the worm body cannot be treated as a linear or constant force generator in biomechanics modeling.

The micropillar-based force measurement system can be readily extended to other applications. One example is to fill the channels with water and investigate the force patterns for C. elegans swimming [28], [29], which is another important yet little-known locomotion different from the wellunderstood crawling on substrate surface. Another example is to explore the configuration of pillars so that they may enhance the locomotion of C. elegans as reported in [30], where the speed of wild-type C. elegans was improved 10 times due to the structured and short agar pillars. If the enhanced locomotion can be observed for PDMS pillars, using PDMS pillars will provide force measurement which was not otherwise achievable by agar pillars during the motion, permitting a comparison of $C$. elegans forces for different pillar structures. The results are likely to explain from the prospective of force how the enhanced locomotion is originated.

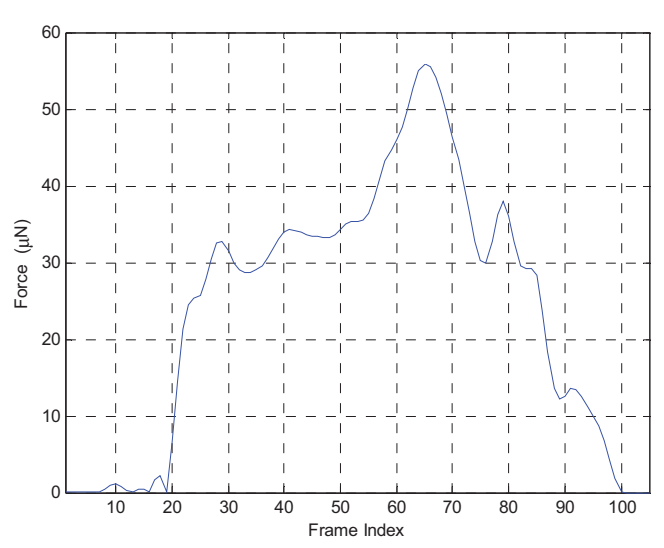

Fig. 10. Force measurement of a selected pillar over the course from initial contact till exit of the contact between the pillar and worm. 
While the data presented here was to picture functionality of our developed automated tracking and force measurement system, it is capable of collecting as much data as desired for more thorough force pattern analysis and locomotion characteristics extraction of $C$. elegans in our future work. This automated approach allows obtaining force patterns for various locomotive behaviors of C. elegans, such as turning, foraging, and forwarding/reversing, and their respective phenotypes. Comparison of these force patterns could help establish the relationship between muscle arms and force patterns of C. elegans in motion, and thus give a better understanding of the genetics controlling muscle arm development.

\section{CONCLUSION}

A novel system consisting of a microfabricated PDMS device to load C. elegans in a matrix of micropillars in a channel, and an image processing algorithm to automatically infer forces from measured micropillar deflections was introduced. It was shown that the image processing algorithm is able to track C. elegans and contact pillars, accurately measure the deflection all automatically. For a worm of $60 \mu \mathrm{m}$ wide, the force resolution is $0.33 \mu \mathrm{N}$. In the example, C. elegans was observed to generate a maximum force of $55.41 \mu \mathrm{N}$ to the micropillars, while having a continuous force output spectrum. The force pattern based on the direction of movement or resistance experienced matches biological implications of muscles. The reported system can be readily combined with existing small-animal screening systems and has the potential to enable highthroughput screening of force patterns in $C$. elegans locomotive phenotypes.

\section{ACKNOWLEDGMENT}

The authors would like to thank Craig Galilee for providing the C.elegans and Helen Devereux for technical assistance. This work was supported by the MacDiarmid Institute for Advanced Materials and Nanotechnology.

\section{REFERENCES}

[1] T. C. e. S. Consortium, "Genome Sequence of the Nematode $C$. elegans: A Platform for Investigating Biology," Science vol. 282, pp. 2012-2018, 1998.

[2] C. Cronin, J. Mendel, S. Mukhtar, Y.-M. Kim, R. Stirbl, J. Bruck and P. Sternberg, "An automated system for measuring parameters of nematode sinusoidal movement," BMC Genetics, 2005.

[3] S.-J. Park, M. B. Goodman and B. L. Pruitt, "Analysis of nematode mechanics by piezoresistive displacement clamp," Proc. Natl. Acad. Sci. U.S.A. vol. 44, pp. 17376-17381, 2007.

[4] L. Herndon, P. Schmeissner, J. Dudaronek, P. Brown, K. Listner, Y. Sakano, M. Paupard, D. Hall and M. Driscoll, "Stochastic and genetic factors influence tissue-specific decline in ageing C. elegans," Nature vol.419, pp. $808-814,2002$.

[5] G. Anderson, W. Boyd and P. Williams, "Assessment of sublethal endpoints for toxicity testing with the nematode Caenorhabditis elegans," Environ. Toxicol. Chem. vol. 20, pp. 833 - 838, 2001.

[6] W. Boyd, R. Cole, G. Anderson and P. Williams ,'The effects of metals and food availability on the behavior of Caenorhabditis elegans," Environ. Toxicol. Chem. vol. 22, pp. 3049-3055, 2003.
[7] W. Boyd and P. Williams, "Comparison of the sensitivity of three nematode species to copper and their utility in aquatic and soil toxicity tests," Environ. Toxicol. Chem. vol. 22, pp. 2768 - 2774, 2003.

[8] S. Brenner, "The genetics of Caenorhabditis elegans," Genetics vol. 77, pp. $71-94,1974$.

[9] D. M. Miller-III, C. J. Niemeyer and P. Chitkara,"'Dominant unc-37 Mutations Suppress the Movement Defect of a Homeodomain Mutation in unc-4, a Neural Specificity Gene in Caenorhabditis elegans," Genetics vol. 135, pp. 741-753, 1993.

[10] L. Brundage, L. Avery, A. Katz, U.-J. Kim, J. E. Mendel, P. W. Sternberg and M. I. Simon, "Mutations in a C. elegans Gq[alpha] Gene Disrupt Movement, Egg Laying, and Viability," Neuron vol. 5, pp. 999-1009, 1996.

[11] T. A. Starich, R. K. Herman and J. E. Shaw, “ Molecular and Genetic Analysis of unc-7, a Caenorhabditis elegans Gene Required for Coordinated Locomotion," Genetics vol. 133, pp. 527-541, 1993.

[12] S. J. Dixon, P.J. Roy, "Muscle arm development in Caenorhabditis elegans," Development, vol. 132, pp. 3079, 2005.

[13] A. Harris, P. Wild and D. Stopak, "Silicone rubber substrata: a new wrinkle in the study of cell locomotion," Science vol. 208, pp. 177179,1980

[14] J. L. Tan, J. Tien, D. M. Pirone, D. S. Gray, K. Bhadriraju and C. S. Chen, "Cells lying on a bed of microneedles: An approach to isolate mechanical force," Proc. Natl. Acad. Sci. U. S. A. vol. 4, pp. 1484$1489,2003$.

[15] O. du Roure, A. Saez, A. Buguin, R. H. Austin, P. Chavrier, P. Siberzan and B. Ladoux, "Force mapping in epithelial cell migration Proc. Natl. Acad. Sci. U. S. A. vol. 7, pp. 2390-2395, 2005.

[16] Y. Zhao and X. Zhang, "Cellular mechanics study in cardiac myocytes using PDMS pillars array," Sensors and Actuators A: Physical, vol. 125, pp. 398-404, 2006.

[17] Y. N. Xia and G. M. Whitesides, "Soft lithography Angew. Chem. Int Edit., vol. 37, pp. 551-575, 1998.

[18] J. C. Doll, N. Harjee, N. Klejwa, R. Kwon, S. M. Coulthard, B. Petzold, M. B. Goodman and B. L. Pruitt, "SU-8 force sensing pillar arrays for biological measurements," Lab Chip, vol. 9, pp. 1449-1454, 2009

[19] A. Ghanbari, V. Nock, W. Wang, R. Blaikie, J. G. Chase, C. XiaoQi and C. E. Hann, "Force Pattern Characterization of C. elegans in Motion," Proc. 15th International Conference on Mechatronics and Machine Vision in Practice, pp. 634-639, 2008

[20] X. Liu, Y. Sun, W. Wang and B. M. Lansdorp, "Vision-based cellular force measurement using an elastic microfabricated device," $J$. Micromech. Microeng., vol. 17, pp. 1281-1288, 2007.

[21] W. Wang, Y. Sun, S.J. Dixon, M. Alexander, and P.J. Roy, "An automated micropositioning system for investigating C. elegans locomotive behavior," Journal for the Association for Laboratory Automation, vol. 14, no. 5, pp. 269-276, 2009.

[22] P. Soille, Morphological Image Analysis: Principles and Applications. New York: Springer-Verlag, pp. 173-174, 1999.

[23] P. V. C. Hough, A method and means for recognizing complex patterns, U. S. Patent No.3.069.654, 1962.

[24] C. Kimme, D. Ballard, J. Sklansky, "Finding circles by an array of accumulators," Comm., ACM, vol. 18, pp. 120-122, 1975.

[25] R. O. Duda, P. E. Hutt, "Use of Hough transformation to detect lines and curves in pictures," Comm., ACM, vol. 15, pp. 11-15, 1972.

[26] F. O'Gorman, and M.B. Clowes, "Finding picture edges through collinearity of feature points," Proc. Third Internat. Joint Conf. on Artifi. Intel., pp. 543-555, 1973

[27] J. Gray, H. W. Lissmann, "The locomotion of nematodes," J Exp Biol, vol. 41, pp. 135-154, 1964.

[28] J. T. Pierce-Shimomura, B. L. Chen, J.J. Mun, R. Ho,R. Sarkis, and S. L. McIntire, "Genetic analysis of crawling and swimming locomotory paterterns in C. elegans," Proc. Natl. Acad. Sci. U. S. A., vol. 52, pp. 20982-20987, 2008

[29] S. Berri, J.H. Boyle, M. Tassieri, I.A. Hope, and N. Cohen, "Forward locomotion of the nematode $C$. elegans is achieved through modulation of a single gait," HFSP Journal, vol. 3, pp. 186-193, 2009.

[30] S. Park, H. Hwang, S.-W. Nam, F. Martinez, R. H. Austin and W. S. Ryu, "Enhanced Caenorhabditis elegans locomotion in a structured microfluidic environment," PLOS ONE 36 e2550, 2008. 